\title{
Breves consideraciones sobre la asistencia médica forzosa a los internos en los centros penitenciarios*
}

\author{
J E Arribas López
}

Jurista del Cuerpo Superior de Técnicos de Instituciones Penitenciarias Secretaría General de Instituciones Penitenciarias

\section{RESUMEN}

En una sentencia de octubre de 2005 la Audiencia Nacional, declara la responsabilidad patrimonial del Ministerio del Interior (Dirección General de Instituciones Penitenciarias), por apreciar una concurrencia de culpas entre un interno fallecido en prisión y la Administración, ya que, aunque observa que fue correcta la actuación de los servicios médicos penitenciarios en el reconocimiento, diagnóstico y seguimiento de las enfermedades que padecía, antes y durante su internamiento, sin embargo tuvieron que adoptar, ante las reiteradas negativas de aquél a someterse a la medicación prescrita, las medidas reglamentarias oportunas con la correspondiente autorización de la dirección del centro penitenciario, a fin de que se cumpliera el tratamiento indicado. Sentada la indiscutible responsabilidad de la Administración de actuar, velando por la vida, integridad y salud de los internos cuando se observa un riesgo directo e inmediato utilizando la fuerza si es preciso, cuando se trata de procesos patológicos de curso prolongado, que puede llevar a una limitación de los derechos del interno más allá de lo necesario y someterlo a la aplicación habitual de unas medidas de fuerza que están concebidas y reguladas sólo para situaciones excepcionales.

Palabras clave: Pacientes Desistentes del Tratamiento, Legislación \& Jurisprudencia, Rol Judicial, Prisiones.

\section{SOME BRIEF COMMENTARIES ABOUT ENFORCED MEDICAL CARE FOR PRISON INMATES}

\begin{abstract}
A decision by the National Court in October 2005 declared that the Ministry of the Interior (General Directorate of Prisons) held pecuniary liability as a result of a concurrence of guilt between a deceased prison inmate and the prison Administration. It was observed that the prison health care staff had acted correctly when checking, diagnosing and monitoring the illness of the patient before and during admission. However, when faced with repeated refusals by the inmate to accept the prescribed treatment, they had to take suitable regulatory measures to comply with the terms of the indicated treatment with authorisation from the prison director. The Administration has a clearly established and undeniable liability to care for the life, integrity and health of prison inmates when a direct and immediate risk is observed, using force if necessary to deal with prolonged pathological conditions. This may bring about restrictions of the inmate's rights beyond those considered to be strictly necessary and lead to him/her being subject to the use of enforcement measures that are conceived of and designed for purely exceptional circumstances.
\end{abstract}

Key words: Patient Dropouts, Legislation \& Jurisprudence, Judicial Role, Prisons.

Texto recibido: enero 2009

Texto aceptado: mayo 2009

"Este artículo fue publicado en la Revista Actualidad Jurídica ARANZADI. AÑO XVI, No 705 de 18 de mayo de 2006. El Consejo de redacción de la RESP ha estimado conveniente su publicación para extender su difusión en un ámbito de interés no cubierto por aquella revista. 


\section{INTRODUCCIÓN}

La Sentencia de 18 de octubre de 2005 de la Sala de lo Contencioso-Administrativo del Tribunal Supremo, más allá del caso concreto resuelto, supone, a mi modo de ver, el que se esté en vías de alcanzar un estadio muy avanzado, quizá en exceso avanzado, en la consideración del deber de las Instituciones penitenciarias de velar por la vida, integridad y salud de los internos, en los supuestos en que éstos se nieguen a recibir tratamiento médico adecuado a las dolencias que padezcan.

Muy resumidamente, la resolución judicial reseñada, casando la Sentencia previa de la Sala de lo Contencioso-Administrativo de la Audiencia Nacional, declara la responsabilidad patrimonial del Ministerio del Interior (Dirección General de Instituciones Penitenciarias), modulando la indemnización a abonar a los demandantes y fijándola en 12.020,24 euros, por apreciar una concurrencia de culpas entre el propio interno fallecido y la Administración, ya que, aunque observa que fue correcta la actuación de los servicios médicos penitenciarios en el reconocimiento, diagnóstico y seguimiento de las enfermedades que padecía, antes y durante su internamiento, sin embargo $t u-$ vieron que adoptar, ante las reiteradas negativas de aquél a someterse a la medicación prescrita, las medidas reglamentarias oportunas con la correspondiente autorización de la dirección del centro penitenciario, a fin de que se cumpliera el tratamiento indicado; aprecia el tribunal, por tanto, entre la actuación administrativa y el daño una relación de causalidad, una conexión mínima, indirecta de causa y efecto, sobrevenida y concurrente con el comportamiento de la propia víctima. En su razonamiento trae a colación, primero, lo dispuesto en el artículo 3.4 de la Ley Orgánica 1/1979, de 26 de septiembre, General Penitenciaria (en adelante, LOGP), relativo al deber de la Administración penitenciaria de velar por la vida, integridad y salud de los internos y, segundo, la posibilidad de aplicación de medios coercitivos ${ }^{1}$ para evitar daños de los internos a si mismos.

El objetivo de este comentario, reconozco que afrentosamente breve para lo que el tema merece por la importantísima carga axiológica en él contenida y por las controvertidas cuestiones jurídicas a que puede dar lugar, es llamar la atención sobre algunos puntos que considero especialmente problemáticos en el manejo generalizado de la doctrina que irradia la Sentencia de 18 de octubre de 2005: la aplicación en el seno de las Instituciones penitenciarias de tratamiento médico en contra de la voluntad del recluso, utilizando, incluso, los medios coercitivos previstos en su legislación reguladora.
Aun en unas notas tan exiguas como éstas, antes de poner de relieve esos aspectos que pueden resultar controvertidos, es conveniente centrar el tema y, para ello, es imprescindible hacer una mera alusión, primero, a las normas básicas que, en el ámbito penitenciario, pueden sustentar la aplicación de un tratamiento médico forzoso a los internados en los establecimientos penitenciarios y que, desde un determinado punto de vista, no son sino concreciones positivas de una relación especial de sujeción; y, segundo, a la doctrina que ha emanado del Tribunal Constitucional cuando se han sometido a su consideración supuestos de alguna forma similares.

\section{LA RELACIÓN JURÍDICA PENITENCIARIA: EL DEBER DE VELAR POR LA VIDA, INTEGRIDAD Y SALUD DE LOS INTERNOS Y LA UTILIZACIÓN DE MEDIOS COERCITIVOS}

La Sentencia a ejecutar, si es que impone una condena de prisión, o el auto de prisión preventiva a cumplimentar, constituyen el acta fundacional de una relación jurídica peculiar entre el Estado y el ciudadano que ingresa, penado o preso, en un establecimiento penitenciario. La singularidad de este vínculo teórico, abstracto, se materializa en un entramado de derechos y deberes recíprocos interno-Administración penitenciaria, contemplado básicamente en la legislación penitenciaria, inexistente en el caso de los ciudadanos que no están encarcelados. Así, por ejemplo, una persona no sometida a una pena de prisión o a una medida cautelar de prisión preventiva no tiene, obviamente, el deber jurídico de permanecer en el establecimiento ${ }^{2}$ ni el de acatar las normas de régimen interior del $\mathrm{mismo}^{3}$; por el contrario, tampoco tiene el derecho a que la Administración penitenciaria vele por su vida, integridad y salud ${ }^{4}$ o a que le proporcione una alimentación adecuada ${ }^{5}$.

La ligazón jurídica que une al interno con el Estado representado en la Administración penitenciaria, por cuanto es distinta y se superpone a la general que enlaza a cualquier otro ciudadano con aquél, puede encuadrarse sin ninguna dificultad en la categoría de relaciones especiales de sujeción; es más, junto a la que vincula a los funcionarios públicos con la Administración a la que sirven, constituye aquélla uno de sus ejemplos más paradigmáticos. En este sentido, es importante indicar que el Tribunal Constitucional $^{6}$, a pesar de dejar constancia de la imprecisión del concepto ${ }^{7}$, tiene declarado que no puede ponerse en duda que la reclusión en un centro pe- 
nitenciario origina una relación jurídica especial de sujeción ${ }^{8}$.

Bien, de la normativa penitenciaria que regula esa especial relación jurídica y para el tema que nos ocupa, es necesario volver sobre dos aspectos concretos. Uno de ellos lo acabo de utilizar «ad exemplum» y es el deber que incumbe a las Instituciones penitenciarias de velar "por la vida, integridad y salud de los internos»" ${ }^{9}$ y el otro es la aplicación de los medios coercitivos prevista en el artículo 45 de la misma Ley.

Que la Administración penitenciaria esté obliga$\mathrm{da}$ a proteger la vida, integridad y salud de los internos tiene una proyección palmaria en muchos aspectos de la normativización del internamiento; así, sin intención alguna de ser exhaustivo, desde la proscripción de los malos tratos de palabra u obra ${ }^{10}$ hasta la especial atención prestada al aislamiento en celda como sanción disciplinaria en cuanto puede ser incompatible con el estado de salud física y mental de los internos ${ }^{11}$, pasando por la concreta regulación de la asistencia sanitaria ${ }^{12}$, toda la Ley está impregnada de una u otra forma de la idea motriz de la responsabilidad que incumbe al Estado de velar por la vida, integridad y salud de quienes están sometidos a su custodia en un establecimiento penitenciario.

Aparte del supuesto que ha dado pie a este trabajo, ese especial deber de cuidado, esa peculiar posición de garante, ha sido invocada al declarar los tribunales la responsabilidad patrimonial de la Administración, o su responsabilidad civil subsidiaria, por determinados hechos acaecidos en el interior de los centros penitenciarios. Verbigracia, en el caso de fallecimientos de internos causados por agresiones de compañeros de internamiento con armas blancas de fabricación carcelaria, se reprocha a la Administración, y por eso se declara su responsabilidad, el incumplimiento del deber de impedir, mediante las actividades de control adecuadas (cacheos, registros, requisas), que los agresores tuvieran a su disposición aquellos instrumentos con los que finalmente causaron la muerte de otro recluso.

El artículo 45 de la LOGP se refiere a la utilización de lo que ese mismo precepto denomina como medios coercitivos; se trata, ni más ni menos, que de la regulación de determinados casos de ejercicio de la fuerza, de la violencia legítima por parte del Estado en el interior de las prisiones. No creo que resulte necesario detenerme sobre la imperiosa necesidad que surge en algunas ocasiones de emplear, tanto en la «vida libre» como en los recintos penitenciarios, la fuerza mínima imprescindible o la coacción necesaria con el objetivo de evitar las consecuencias o efectos indeseados que pueden derivarse de algunas omisiones o acciones, a veces muy agresivas y violentas, protagonizadas, en nuestro caso, por internos.

La LOGP establece, en el número 1 del artículo 45, que sólo podrán utilizarse, con autorización del director, órgano superior en la jerarquía administrativa del centro, aquellos medios coercitivos que se establezcan reglamentariamente en los siguientes casos: para impedir actos de evasión o violencia de los internos; para evitar daños de los internos a sí mismos, o a otras personas o a cosas; $y$, finalmente, para vencer la resistencia activa o pasiva de los internos a las órdenes del personal penitenciario en el ejercicio de su cargo.

También contempla la Ley ${ }^{13}$ que si la urgencia de la situación no ha permitido recabar la previa autorización del director para el empleo de los medios coercitivos previstos, debe comunicársele inmediatamente; a su vez, éste, el director, debe poner su utilización en conocimiento del juez de vigilancia, órgano jurisdiccional previsto en la propia LOGP que, entre las importantísimas funciones contempladas en su artículo 76, tiene la de «salvaguardar los derechos de los internos y corregir los abusos y desviaciones que en el cumplimiento de los preceptos del régimen penitenciario puedan producirse».

Finalmente, la LOGP establece que el uso de las medidas coercitivas estará dirigido exclusivamente al reestablecimiento de la normalidad y sólo subsistirá el tiempo estrictamente necesario ${ }^{14}$.

Por su parte, el Reglamento Penitenciario, aprobado por Real Decreto 190/1996, de 9 de febrero (en adelante, RP), detalla en el artículo 72.1 que son medios coercitivos los siguientes: el aislamiento provisional, la fuerza física personal, las defensas de goma, los aerosoles de acción adecuada y las esposas. En el mismo precepto, enuncia una serie de principios que deberán ser tenidos en cuenta en su utilización: su uso será proporcional al fin pretendido, nunca supondrán una sanción encubierta y sólo se aplicarán cuando no exista otra manera menos gravosa para conseguir la finalidad perseguida y por el tiempo estrictamente necesario.

Bien, si, por un lado, la Administración penitenciaria debe salvaguardar la vida, integridad y salud de los internos ${ }^{15}$, colocándose así en una posición de garante y, por otro, si está legitimada para emplear los medios coercitivos necesarios para evitar daños de los internos a sí mismos ${ }^{16}$, se puede colegir que incurriría en responsabilidad si, ante acciones u omisiones de los propios internos que pueden poner en peligro su vida, su integridad física o su salud, no actúa evitando el resultado, sobreponiéndose, incluso por la fuer$\mathrm{za}$, a la propia voluntad del interno. 
El juicio sobre la adecuación o la reprochabilidad de la actuación administrativa no tiene mayores dificultades ante determinadas contingencias. Así, por ejemplo, frente ante maniobras claramente autolesivas o comportamientos inequívocamente autolíticos de los internos (piénsese en un intento de ahorcamiento, en la ingestión de cuerpos extraños o en heridas producidas con objetos cortantes) debe reaccionarse de forma inmediata con el empleo, incluso, de medidas de fuerza. La consecuencia directa en estos casos de un eventual comportamiento omisivo de la Administración sería el fallecimiento o la producción de lesiones muy graves en el interno.

La solución que se ha dado en casos, digamos, no tan claros, en los que el daño, siendo al final inequívoco o incluso irreversible, no provenía o podía provenir de una acción rápida o instantánea, sino de una actitud más o menos prolongada en el tiempo, nos lleva de lleno al epígrafe siguiente.

\section{SÍNTESIS DE LA DOCTRINA DEL TRIBUNAL CONSTITUCIONAL SOBRE LA ALIMENTACIÓN Y EL TRATAMIENTO MÉDICO FORZOSO EN CASOS DE HUELGA DE HAMBRE}

El supuesto de hecho subyacente en las Sentencias del Tribunal Constitucional en las que se aborda tan trascendente cuestión se refería a internos pertenecientes a la organización terrorista GRAPO. Ante determinadas directrices de política penitenciaria y para exigir su reagrupamiento en un centro penitenciario, los reclusos pertenecientes a la organización se declararon en huelga de hambre; dada su prolongación en el tiempo, la actuación administrativa dio origen, básicamente, resumiendo mucho y haciendo abstracción de matices, a dos tipos de resoluciones judiciales. Las primeras, llegado el caso de que la vida del huelguista corriese peligro cierto, autorizaban la asistencia médica obligatoria y la alimentación forzosa incluso en contra de su voluntad y utilizando medios coercitivos, quedando descartada, no obstante, la alimentación por vía bucal; las segundas sólo la permitían cuando el interno perdiese la consciencia, impidiéndola consecuentemente hasta entonces. Por la representación de los internos, en el caso de los primeros pronunciamientos y por el Ministerio Fiscal, en el de los segundos, se presentaron sendos recursos de amparo que determinaron la intervención del Tribunal Constitucional.

Siendo del todo injusto con el valioso despliegue argumentativo del Tribunal, quizá los siguientes puntos puedan servir de resumen de su posición, que centro, fundamentalmente, en la Sentencia 120/1990, de 27 de junio, y en el análisis que realiza sobre si el tratamiento médico y la alimentación forzosa vulneran el artículo 15.1 de la Constitución Española de 1978 (en adelante, CE), en tanto reconoce el derecho a la vida y a la integridad física y moral y proscribe la tortura y las penas o tratos inhumanos o degradantes.

- El Tribunal destaca que la intervención médica forzosa, por los valores humanos que en ella se implican, es un tema de excepcional importancia que irradia sus efectos a distintos sectores del ordenamiento jurídico, especialmente al constitucional y al penal; más allá del Derecho, hay en juego concepciones distintas de la vida humana.

- La decisión que el Tribunal Constitucional analiza es la intervención médica mínima indispensable para conseguir el fin constitucional que la justifica y que la permite tan sólo en el momento en que, según, la ciencia médica, corra «riesgo cierto» la vida del recluso y en la forma que el juez de vigilancia penitenciaria determine, prohibiendo que se suministre alimentación bucal en contra de la voluntad consciente del interno. En resumidas cuentas, lo que se estudia es si la alimentación forzosa vía parenteral, cuando, según los conocimientos médicos, sea necesaria para evitar el riesgo de muerte, es respetuosa con los derechos fundamentales de los internos.

- Para el Tribunal, la asistencia médica se impone en el marco de la relación especial de sujeción que vincula a los internos con la Administración penitenciaria; ésta, en virtud de tal situación especial, viene obligada a velar por la vida, integridad y salud de los internos sometidos a su custodia. Este deber «le viene impuesto por el artículo 3.4 de la LOGP, que es la Ley a la que se remite el artículo 25.2 de la Constitución como la habilitada para establecer limitaciones a los derechos fundamentales de los reclusos, y que tiene por finalidad, en el caso debatido, proteger bienes constitucionalmente consagrados, como son la vida y la salud de las personas». Conviene recordar ahora que el artículo 25.2, después de fijar la orientación de las penas privativas de libertad y de las medidas de seguridad, proclama que el condenado a pena de prisión que estuviere cumpliendo la misma "gozará de los derechos fundamentales de este Capítulo, a excepción de los que se vean expresamente limitados por el contenido del fallo condenatorio, el sentido de la pena y la ley penitenciaria» (la cursiva es mía).

- La asistencia médica obligatoria para evitar el peligro de muerte se manifiesta como un medio imprescindiblemente necesario para evitar la pérdida del 
bien de la vida de los internos, que el Estado tiene obligación legal de proteger, acudiendo, en último término, a dicho medio coactivo, al menos si se trata de presos declarados en huelga de hambre reivindicativa cuya finalidad no es la pérdida de la vida. Razona el Tribunal que la negativa a recibir asistencia médica sitúa al Estado, en forma arbitraria, ante la injusta situación de modificar una decisión de política penitenciara, que es legítima mientras no sea anulada judicialmente, o contemplar la muerte de personas que están bajo su custodia y cuya vida está legalmente obligado a preservar y proteger.

- Que para efectuar la intervención médica se permita el empleo de medios coercitivos no es determinante, ya que no es la coercitividad de trato más allá de lo proporcionado, sino su desmedida severidad, su innecesario rigor y su carácter vejatorio lo que a los efectos de la prohibición constitucional resulta relevante.

- Además de la vulneración del artículo 15 de la Constitución, también descarta su más alto intérprete la de los artículos 16.1 (libertad ideológica), 17.1 (libertad personal) y 18.1 (intimidad personal).

\section{PROBLEMÁTICA PLANTEADA}

Como dije al principio, la generalización de la doctrina emanada de la Sentencia de 18 de octubre de 2005, de la Sala Tercera del Tribunal Supremo, presenta, a mi modo de ver, aspectos problemáticos que, sin intención alguna de exhaustividad, me propongo meramente poner de manifiesto a continuación. El trazado argumental de la resolución judicial, normativamente hablando, queda cimentado en los artículos 3.4 y 45 de la LOGP: la Administración penitenciaria tiene el deber de velar por la vida, integridad y salud de los internos, siendo necesario para ello que aplique el tratamiento médico prescrito, incluso utilizando los medios coercitivos previstos para vencer la posible voluntad contraria del interno a recibirlo. Como no se procedió de esta forma, la actuación administrativa no se considera adecuada, su culpa concurre con la del propio interno fallecido y se declara su responsabilidad patrimonial.

El planteamiento del Tribunal Supremo, en el fondo, descansa, «servata distantia», en las mismas bases jurídicas y normativas que el Tribunal Constitucional al resolver los recursos de amparo aludidos: $r e-$ lación especial de sujeción interno-Administración penitenciaria, regulada por normas (Ley Penitenciaria) que pueden limitar derechos reconocidos a las personas no privadas de libertad; deber de la última de velar por la vida, integridad y salud de los internos, con lo cual se sitúa en una posición de garante; y, por último, utilización de los medios coercitivos previstos para evitar daños de los internos a sí mismos.

Ahora bien, desde mi punto de vista, aun con los mismos cimientos de Derecho positivo, es discutible que la doctrina del Tribunal Constitucional sea aplicable sin más a supuestos como el resuelto por el Supremo, porque, entre otras cosas, bien se encarga el primero de advertir en varias ocasiones que su resolución se ciñe al concreto caso planteado, limitando al mismo sus consideraciones «con el fin de evitar todo confusionismo con otros supuestos de asistencia médica obligatoria distintos del presente, que quizás requieran diferente solución en atención a las diferentes condiciones en que aquéllos se susciten» (STC 120/1990, de 27 de junio, FJ 5º la cursiva es mía); insistiendo en la misma idea al manifestar que la imposición de limitaciones a los derechos fundamentales de internos que se colocan en peligro de muerte a consecuencia de una huelga de hambre reivindicativa "podrían resultar contrarias a esos derechos si se tratara de ciudadanos libres o incluso internos que se encuentren en situaciones distintas» (FJ 6; también ahora la cursiva es mía).

Además de las propias advertencias del Tribunal Constitucional, hay demasiados e importantes aspectos diferenciales entre los casos sometidos a su consideración y otros de los que puede ser representativo el resuelto por el Supremo en la Sentencia de 18 de octubre de 2005, como para entender aplicable su doctrina a éstos. Una de las diferencias que pueden observarse entre ambos casos es que, en el resuelto por este último, los procesos patológicos del interno fueron muy prolongados en el tiempo y en su curso se jalonaron diferentes negativas a someterse a la medicación prescrita e, incluso, en algún momento, a realización de analíticas. Consecuentemente, resulta casi imposible identificar un momento dado en el que pudiera apreciarse un riesgo vital inmediato directamente derivado de su negativa a someterse a la medicación pautada. Teniendo esto en cuenta, y a la vista de lo resuelto, para que la valoración del proceder de los servicios penitenciarios hubiese sido del todo correcta (no olvidemos, como consigné al principio, que el Tribunal reconoce que lo fue en el reconocimiento, diagnóstico y seguimiento de las enfermedades) y no merecedora de la imputación de una culpa concurrente con la del interno -origen de la responsabilidad patrimonial declarada- habría sido necesario hacer caso omiso a su voluntad en las ocasiones en que se negó a tomar la medicación y utilizar, en caso necesario, los medios coercitivos previstos para vencer 
su resistencia; y esto, obsérvese, insisto, en enfermedades de curso muy largo que exigen tratamientos médicos y farmacológicos prolongados en el tiempo.

La Ley 41/2002, de 14 de noviembre, básica reguladora de la autonomía del paciente y de derechos y obligaciones en materia de información y documentación clínica (en adelante, Ley 41/2002), establece, en el ámbito de los principios generales, en su artículo 2.4, lo que sigue: «Todo paciente o usuario tiene derecho a negarse al tratamiento, excepto en los casos determinados por la Ley». Más adelante, en el Capítulo IV, dedicado específicamente al respeto a la autonomía del paciente, dice el artículo 8 que toda actuación en el ámbito de la salud de un paciente «necesita el consentimiento libre y voluntario del afectado, una vez que, recibida la información prevista en el artículo 4 [que regula el derecho a la información asistencial], haya valorado las opciones propias del caso».

No obstante lo anterior, el artículo 9.2 autoriza a los facultativos para llevar a cabo las intervenciones clínicas indispensables a favor de la salud del paciente, sin necesidad de contar con su consentimiento cuando, primero, «existe riesgo para la salud pública a causa de razones sanitarias establecidas por la Ley»y, segundo, cuando «existe riesgo inmediato grave para la integridad física o psíquica del enfermo y no es posible conseguir su autorización [...]». El apartado 3 del mismo artículo regula el consentimiento por representación en tres casos, a saber: cuando el paciente no sea capaz de tomar decisiones o su estado psíquico o físico no le permita hacerse cargo de la situación, cuando esté incapacitado legalmente y cuando el paciente menor de edad no sea capaz intelectual ni emocionalmente de comprender el alcance de la situación.

No cabe duda de que tratar de cohonestar todo lo estipulado en la Ley 41/2002 con supuestos como el que ahora nos ocupa llevaría irremisiblemente a entender que los internos aquejados de enfermedades graves en las que se pudiera pronosticar un desenlace fatal aunque su producción no fuese ni inmediata ni siquiera cercana en el tiempo, no gozarían de los derechos que, relacionados con su autonomía como pacientes, se reconocen al resto de ciudadanos, y ello en virtud del deber de la Administración de velar por su vida, integridad y salud que, de esta forma, se sobrepone y tiene supremacía sobre aquéllos; en definitiva, se produciría una limitación de los derechos del interno por la relación especial de sujeción que le vincula con la Administración penitenciaria.

Tomando en consideración todo lo expuesto, y muy esquemáticamente, los aspectos que considero más discutibles en la aplicación de la doctrina de la tan repetida ya Sentencia de 18 de octubre de 2005 vie- nen definidos, a mi modo de ver, por los siguientes parámetros:

- La existencia de enfermedades de curso prolongado -prevalentes algunas entre la población penitenciaria (pensemos en el VIH-SIDA, en la tuberculosis) - en las que parece difícil, por no decir imposible, la fijación de un momento en el cual la negativa a recibir medicación pueda considerarse causante de un riesgo vital inmediato; se trata más bien de procesos, no de momentos.

- Directamente derivado de lo anterior, la prescripción de tratamientos médicos y/o farmacológicos prolongados en el tiempo que exigen, en ocasiones, tomas diarias y la necesidad subsiguiente, llegado el caso, de forzar su administración, incluso empleando medios coercitivos. Debe dejarse constancia de la circunstancia de que la medicación, en algunos casos, se debe administrar por vía bucal.

- La negación a los internos, en estos supuestos, de sus derechos como pacientes en virtud de la relación especial de sujeción a la que están sometidos.

- La dificultad de aplicación directa de la doctrina emanada del Tribunal Constitucional cuando ha tenido la ocasión de pronunciarse sobre casos que guardan alguna relación con el que es objeto de análisis.

Para finalizar, y como apresurada conclusión, quiero insistir en hacer patente el hecho de que, sentada y asumida la indiscutible responsabilidad de la Administración de actuar, reactiva y proactivamente, velando por la vida, integridad y salud de los internos cuando se observa un riesgo directo e inmediato (antes he puesto algunos ejemplos), utilizando la fuerza si es preciso, en procesos patológicos de curso prolongado (antes he aludido al sida y a la tuberculosis, pero también puede pensarse en otras enfermedades tales como el cáncer necesitado de quimio o radioterapia), la posible sublimación de ese deber puede arrastrarlo al terreno del paroxismo, trayendo como consecuencia, primero, una limitación de los derechos del interno más allá de lo necesario y de lo que cabría entender como razonable y proporcional a su situación de parte en una relación especial de sujeción y, segundo, someterlo, en supuestos no por extremos menos imaginables, a la aplicación habitual de unas medidas de fuerza que están concebidas y reguladas sólo para situaciones excepcionales.

\section{CORRESPONDENCIA}

Talleres Editoriales Cometa, S.A.

Ctra. Castellón, km. 3,400.

50013 Zaragoza. 


\section{REFERENCIAS BIBLIOGRÁFICAS}

1. Ley Orgánica General Penitenciaria $1 / 1979$ de 26 de septiembre, Artículo 45. (B.O.E. número 239, de 05/10/79).

2. Ley Orgánica General Penitenciaria $1 / 1979$ de 26 de septiembre, Artículo 4.1.a. (B.O.E. número 239, de 05/10/79).

3. Ley Orgánica General Penitenciaria $1 / 1979$ de 26 de septiembre, Artículo 4.1.b. (B.O.E. número 239, de 05/10/79).

4. Ley Orgánica General Penitenciaria 1/1979 de 26 de septiembre, Artículo 3.4. (B.O.E. número 239, de 05/10/79).

5. Ley Orgánica General Penitenciaria $1 / 1979$ de 26 de septiembre, Artículo 21.2. (B.O.E. número 239, de 05/10/79).

6. Sentencia del Tribunal Constitucional 120/1990, de 27 de junio.

7. Sentencia del Tribunal Constitucional 61/1990, de 29 de marzo.

8. Sentencia del Tribunal Constitucional 2/1987, de 21 de enero.
9. Ley Orgánica General Penitenciaria 1/1979 de 26 de septiembre, Artículo 3.4. (B.O.E. número 239, de 05/10/79).

10. Ley Orgánica General Penitenciaria $1 / 1979$ de 26 de septiembre, Artículo 6. (B.O.E. número 239, de $05 / 10 / 79$ ).

11. Ley Orgánica General Penitenciaria 1/1979 de 26 de septiembre, Artículo 43. (B.O.E. número 239, de 05/10/79).

12. Ley Orgánica General Penitenciaria 1/1979 de 26 de septiembre, Artículo 36-40. (B.O.E. número 239, de 05/10/79).

13. Ley Orgánica General Penitenciaria 1/1979 de 26 de septiembre, Artículo 45.2. (B.O.E. número 239, de 05/10/79).

14. Ley Orgánica General Penitenciaria $1 / 1979$ de 26 de septiembre, Artículo 45.3. (B.O.E. número 239, de 05/10/79).

15. Ley Orgánica General Penitenciaria 1/1979 de 26 de septiembre, Artículo 3.4. (B.O.E. número 239, de 05/10/79).

16.Ley Orgánica General Penitenciaria 1/1979 de 26 de septiembre, Artículo 45.1.b. (B.O.E. número 239, de 05/10/79). 